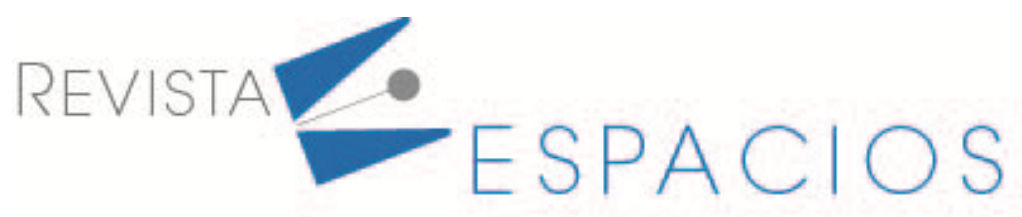

\title{
Estilos gerenciales en función del empoderamiento de los colaboradores de la organización
}

\section{The management style in funtion to empowerment fo the organization workers}

\author{
TREJOS-GIL, Carlos A. ${ }^{1}$ \\ MERIÑO Córdoba, Victor $\mathrm{H}^{2}$ \\ LOPEZ Matheus, Angélica M. ${ }^{3}$ \\ MONCINI-Marrufo, Renata del C. ${ }^{4}$ \\ MARTÍNEZ De Meriño, Carmen Y. ${ }^{5}$ \\ ORTíz Sánchez, Luz M. ${ }^{6}$
}

\section{Resumen}

El presente trabajo pretende identificar las características de las empresas que aplican empoderamiento en el área administrativa, relacionando así la incidencia que tiene los diferentes estilos gerenciales en el empowerment. Con metodología cuantitativa y análisis exploratorio, se estudiaron 28 empresas las cuales evidenciaron resultados concluyentes en aspectos como participación, gerenciales, autoritarios y de resolución de conflictos. Se concluye que en las empresas se aplica el estilo gerencial democrático, consultivo, participativo y colegiado generando un ambiente propicio para el empoderamiento.

Palabras clave: empoderamiento, empresa, toma de decisiones, participación de los trabajadores

\begin{abstract}
This work was to propose identify business caracteristics whit empowerment in teh administration area, relationated the incidence the diferent management styles in the empowerment. Cuantitative method and exploratorys analysis, 28 business revelated it concluyent results in particpation, manegement, authority and conflict resolution aspects. it conclude the study that companyes applied democratic style manegement, consultive, participative and colegied, cause appropiated enviroment to empowerment.
\end{abstract}

key words: empowerment, business, decision making, workers participation

\footnotetext{
${ }^{1}$ Magister en Dirección Estratégica (Especialidad gerencia); MBA - Máster Internacional en Administración y Dirección de Empresas (MBA); especialista en Alta Gerencia; profesional en Publicidad; docente investigador de la Facultad de Comunicación Publicidad y Diseño; integrante del grupo de investigación URBANITAS de la Universidad Católica Luis Amigó. https://orcid.org/0000-0002-6769-3396 Correo: carlos.trejosgi@amigo.edu.co 2 Ph.D. en Educación. Magister en Educación. Licenciado en Matemática. Investigador Senior. Docente investigador de la Facultad de Ciencias Administrativas, Económicas y Contables. Grupo de Investigación GORAS de la Universidad Católica Luis Amigó. Medellín - Colombia. https://orcid.org/0000-0001-8911-72024 Correo: victor.merinoco@amigo.edu.co

${ }^{3}$ Licenciada en Administración mención Gerencia y Mercadeo. Especialista en Gerencia de Recursos Humanos. Correo electrónico: A.lopezmatheus@gmail.com.Venezuela.

${ }^{4}$ Licenciada en Administración mención Gerencia Industrial y Licenciada en Contaduría Pública. Magister Scientiarum en Gerencia Financiera. Docente de la Universidad Alonso de Ojeda. Correo electrónico: rmmarrufo@gmail.com. Venezuela

${ }^{5}$ Ph.D. en Educación. Magister en Educación. Licenciada en Comercio. Investigadora Senior. Docente investigadora de la Facultad de Educación y Humanidades. Grupo de investigación GINCIS de la Universidad Católica Luis Amigó. Medellín - Colombia. https://orcid.org/0000-0002-8134-2306 Correo: carmen.martinez@amigo.edu.co

${ }^{6}$ Especialista en Docencia Investigativa Universitaria. Contadora Pública. Docente de la Facultad de Ciencias Administrativas, Económicas y Contables de la Universidad Católica Luis Amigó. Correo: luz.ortizsa@amigo.edu.co.
} 


\section{Introducción}

Mundialmente han ocurrido inumerables cambios que originan transformaciones en el entorno, como la alta demanda de servicios, la competencia entre organizaciones por ofrecer un mejor producto, el desarrollo de nuevas tecnologías, consolidación de nuevos mercados, posicionamiento de innovaciones tecnológicas, expansión y globalización entre otros; ámbitos que influyen tanto en la efectividad como en la productividad de las empresas que muestran lo poco atractivo y funcional de lo que es tener una organización piramidal, en el que la axiología de los gerentes exponen comportamientos éticos para la gestión de sus empresas (Meriño, et al., 2020) y en el que los directivos, supervisores o coordinadores, no involucran al empleado en los procesos de formulación de objetivos y toma de decisiones.

Por tanto, se hace indispensable el fortalecimiento o empoderamiento de los trabajadores, tanto a nivel personal como en lo profesional, a objeto de poder asumir responsabilidades, retos, funciones delegadas o atribuidas en cada tipo actividad a ejecutar; por cuanto de esta forma, se amplia la oportunidad a los trabajadores de manifestar sus competencias y destrezas. Teniendo en cuenta que las organizaciones constantemente están en contacto con ambientes externos e internos realizando los respectivos monitoreos Trejos-Gil (2008), sobre todo sus colaboradores, el empowerment se vislumbra como un estilo, tanto para capacitar como para facultar a cada persona a fin que tenga poder, autoridad y autodeterminación para decidir sobre la toma de desiciones en su trabajo, sin necesidad de requerir constantemente permiso a sus superiores para actuar; aunque, esto no significa una pérdida de autoridad de la alta gerencia sino un trabajo en equipo donde también actúa la directiva.

\subsection{Empoderamiento como actitud delegada de enfoque gerencial}

El empoderamiento es un recurso del que las empresas en su estado competitivo capacitan a sus colaboradores para que tengan la capacidad de detectar situaciones específicas en las que se pueda tomar desiciones en los diferentes procesos y procedimientos de las empresas; se minimiza la necesidad de requerir soluciones que no requieren de decisión directiva habilitando tiempo para los ejecutivos de la compañía en otos apectos más determinantes (Calderón, 2003). En este sentido, el empowerment cataliza los cambios en el lugar de trabajo y posibilita a las personas que usen su poder personal habilitándolas a ser proactivas, y autodeterminadas (De la Garza, Carpio, López \& Rodríguez, 2020; do Nascimento \& Beuren, 2014; Robbins \& Coulter, 2010, p.132); por ser un proceso en el que se mejora la efectividad y el desempeño en las organizaciones al introducir el cambio significativo en la cultura y el clima organizacional, logrando así, maximizar el potencial de las personas al incrementar su creatividad, responsabilidad, autoridad y compromiso; además de contribuir al impulso del liderazgo y al trabajo en equipo.

No solo se trata de un tipo de "actitud" sino también un aporte sustancial lo tiene el estilo gerencial. El estilo gerencial o de liderazgo, constituye un medio útil para el efectivo cumplimiento del trabajo, es un método aceptable para la determinación del comportamiento de los administradores y propiciar nuevos entendimientos entre los subordinados y sus líderes (De la Garza, et al., 2020; Werther \& Davis, 2013). La fuerza combinada de los conocimientos sobre la necesidad de los empleados y de los cambios que ocurren dentro de las instituciones y los valores sociales, permitió el reconocimiento que existieran mejores maneras de administrar los sistemas organizacionales (Shenav, 1995; Werther \& Davis, 2013).

El Empowerment pretende crear un ambiente en donde los empleados de todos los niveles sienten que tienen una influencia real sobre los estándares de calidad, servicio y eficiencia del negocio dentro de sus actividades y áreas de responsabilidad. Esto genera un involucramiento por parte de los trabajadores para alcanzar las metas de la organización con un sentido de compromiso y autocontrol. Del mismo modo se basa en la capacitación del personal para delegar autoridad, autodeterminación y poderpara tomar desiciones sin requerir la autorización de superiores como suele suceder en las organizaciones con estructuras piramidales 
(Díaz, 2009; Blanchard, Carlos \& Randolph, 2009, p.156) por cuanto al colaborador se le concede suficiente autoridad y responsabilidad, para ejecutar las tareas tal como lo ha planificado la organización.

La teoría fundad anteriormente coincide en considerar el empowerment como coadyudante al desempeño tanto eficiente como eficaz de las actividades de los empleados empoderados, el incremento de la creatividad, disminución de la resistencia al cambio, mejora del desempeño, fomento de una nueva cultura, inspirada en transmitir valores, la iniciativa automática de los roles en el trabajo y la visión clara del negocio.

Ahora bien, con base a lo anterior el empoderamiento, se convierte en un enfoque gerencial por fortalecer a los empleados por medio de una mayor satisfacción, motivación por la ejecución de su trabajo, redundando ello, en el incremento de las responsabilidades, compromiso, mejor creatividad, comunicación, capacidad de adaptación al cambio, por último incentiva el entusiasmo y actitud positiva para desarrollar las funciones, llevándolos así a la consecusión de los objetivos planteadas esencialmente en su plataforma estratégica de negocios.

\subsection{Estilo gerencial}

EL administrador o director general de la compañía tiene ciertos tipos de estilos gerenciales ejercidas dentro de sus propias funciones con el propósito de maximizar los resultados cumplimiento efectivo del trabajo en toda la organización (Chiavenato, 2011, p.78; Codina, 2012, p.88). Según Codina (2012, p.88), refiere que dichos estios pueden ser de naturaleza autocrático, democrático, consultivo, participativo, deliberado, colegiado, resolutivo, conflictivo; en este sentido, el impacto puede variar si se trata de un tipo de negocio no estructurado como el de consultoría gerencial, publicidad creativa; o uno altamente estructurado como una refinería o línea de ensamblaje. Por otro lado se debe identificar los componentes del negocio más vulnerables al estilo gerencial, por lo que estos estilos se tienen en cuenta al momento de definir el perfil del gerente, las que están dadas por los requerimientos de los procesos y principalmente por las características de la gente a ser gerenciada.

El estilo gerencial forma parte de una serie de aspectos filosóficos y culturales, algunos métodos en la toma de desiciones y aspectos de estructura y estrategia (Antoncic \& Hisrich, 2001; De Villiers-Scheepers, 2012) las que determinan las conductas en la organización y el comportamiento de cada gerente. Asi mismo estos aspectos

generan un conjunto de relaciones y consecuencias que afectan la estructura organizacional, prioridades en su filosofía y la calidad de la respuesta inmediata de la organización hacia los públicos objetivos cuyas características se constituyen en la contrapartida del estilo gerencial.

Finalmente, los estilos de liderazgo o estilos gerenciales van directamente ligados en los diferentes comportientos proactivos de los colaboradores, afectándo directamente el empoderamiento en cada uno de sus roles específicos de cada colaborador. Del mismo modo, los estidlos gerenciales varían de acuerdo a los deberes a desempeñar por el mismo gerente, sus responsabilidades y el compromiso filosófico tanto para la realización de sus actividades como para el cumplimiento de las expectativas de sus subalternos. En el presente trabajo investigativo se considera para el estudio de la dimensión estilo gerencial, los indicadores autocrático, democrático, consultivo, participativo, deliberado, colegiado, resolutivo y conflictivo (Codina, 2012).

\section{Estilo gerencial Autocrático}

El estilo autocrático, es quel que en el ejercicio de la dirección empresarial toma las desiciones basados en su poder total administrativo, teniendo en cuenta las diferentes alternativas posibles de acción, con baja tolerancia a la ambigüedad y por último asigna las tareas y orienta a sus subordinados controlando detalladamente las acciones impuestas bajo su dirección (Kreitner \& Kinicki, 2018; Solys, Morales \& Medina, 2016; Suárez \& Pinzón, 2020) en otras palabras, él solo planifica y programa lo que se debe hacer, los demás se limitan a atender dicha planificación. 
De esta manera, con base a la definición anterior, el estilo gerencial autocrático, ocurre, cuando éste, da la orden sin consultar previamente y sólo, espera el cumplimiento cabal de tal orden. Por lo general es una persona dogmática, rígida, imparte órdenes sin conceso, sigue las normas y es eficaz en la toma de desiciones aunque puede alterar la armonía del clima organizacional (Chiavenato, 2007, p.106; Hernández, Muñoz \& Barrios, 2017; Suárez \& Pinzón, 2020); es una persona firme y dirige mediante la habilidad para dar, negar recompensas o castigos. Sin embargo, este tipo de estilo gerencial es recomendable para resolver situaciones emergentes, o cuando los subalternos evidencian poca madurez tanto laboral como emocional.

\section{Estilo gerencial Democrático}

Estilo expuesto por primera vez por Lewin (1951) quien lo denominó como estilo democrático también llamado liderazgo participativo, se caracteriza por la acción participativa de los subordinados, toma de desiciones participativas, habilidad de escucha por parte del directivo, no solo ofrece orientación sino también participación de los colaboradores y de hecho los aníma a participar activamente en la mayoría de las funciones (Bateman, 2011, p.110; Solys, Morales \& Medina, 2016). En conjunto, se definen metas y objetivos, se seleccionan las alternativas posibles, se evalúan las mismas y se decide por consenso cuál es la alternativa mejor a seguir; pero siempre se tiene claro que es el directivo quien tiene la última palabra.

De igual modo, el estilo democrático no impone las soluciones, ofrece diferentes alternativas para la selección conjunta con los colaboradores, fomenta la participación de los colaboradores; para esto es impresindible la habilidad de comunicación asertiva y escucha del directivo, la adecuada construcción del previo plan de trabajo, el control de la tensión en el clima laboral, incentivación del trabajo en equipo orienta constantemente hacia la ayuda y la motivación (Drucker, 2012, p.102; Hernández, Muñoz \& Barrios, 2017; Soto, 2012; Suárez \& Pinzón, 2020; Villava \& Fierro, 2017). De esta forma, el gerente democrático, cataliza las opiniones, trata de encontrar una salida satisfactoria para el grupo, en beneficio de todos los miembros que conforman la empresa.

Ahora bien, con base a los conceptos anteriores, el estilo democrático, es caracterizado por la acción participativa de los trabajadores, ya que cumplen con un conjunto de actividades, las cuales son la definición de metas y objetivos, la selección de posibles alternativas, la evaluación de los procedimientos y toman las decisiones de manera equilibrada. Entre sus rasgos principales, está el empleo de numerosas tácticas de negociación que se convierten en medidas de persuasión para poder crear el consenso entre los miembros del equipo.

\section{Estilo gerencial Consultivo}

El estilo consultivo se caracteriza al gerente por definir los objetivos, las metas o los problemas en compañía y conjuntamente con los colaboradores, teniendo en cuenta sus opiniones haciéndolos partícipes de las alternativas de mejora (Goncalves, 2010, p.107; Mora, 2014), este estilo también realiza las funciones de seleccionar las alternativas, evaluarlas, elegir la mejor, hacer la ejecución y controlar.

Ahora bien, el estilo consultivo facilita la información a los colaboradores, busca interesarse por el equipo de trabajadores; crea un ambiente óptimo para la comunicación; tiene un control en las consultas; genera retroalimentación a su equipo de trabajo; logra una mayor participación en los empleados y mejora las relaciones tanto en el área directiva como en las áreas de los empleados.

\section{Estilo gerencial Participativo}

El estilo participativo de Vroom y Yetton, (1973) se cataloga como estilo emblemático dentro la teoría administrativa como respuesta efectiva ante los desafíos de las organizaciones; desde la dirección se promueve las ideas de los subalternos, la participación y ejecuión de aquellas ideas que coadyuden a la productividad a travez de su participación en sus responsabilidades trasnferidas (Chiavenato, 2012; Illanes \& Esparza, 2015; Prieto, Burgos, García \& Rincón, 2016; Villava \& Fierro, 2017). Cultiva la toma de decisiones de sus subalternos con la finalidad de obtener ideas cada vez más útiles y maduras. 
Así mismo, este estilo se enfoca en la consulta entre los miembros de la organización, en ese momento el gerente se reserva el derecho de tomar una decisión además del control; sin embargo, en equipo se resuelven los problemas, se definen los objetivos y se eligen alternativas con el propósito de obtener la mejor opción para la compañía. Importante que el gerente que se identifique con este estilo por lo general cumple con los cuatro aspectos del liderazgo participativo, primero, Tratamiento de conflictos; segundo, alta motivación; tercero, el esfuerzo común; y cuarto,fomento de la responsabilidad (Harvard Deusto Business Review, 2017)

\section{Estilo gerencial Colegiado}

De acuerdo con Jiménez (2004, p.102), el estilo colegiado, "es donde las funciones se ejercen en conjunto incluyendo el control, sin embargo, este estilo de gerencia solamente es aplicable a algunos tipos de organizaciones, estructuras muy especiales". Por ejemplo una sociedad de profesionales donde cada uno aporta desde su especialidad, disciplina y nadie es jefe de nadie. Ahora bien, en virtud del concepto "Colegiado", la orientación de este estilo gerencial se dirige al trabajo en equipo todas las funciones se ejercen en conjunto; nadie es jefe (Banco Popular - Impulsa [BP-I], 2013), en este estilo colegiado se pretende lograr de los empleados una mayor responsabilidad en el trabajo, significando esto, un reconocimiento tanto para su labor como para la organización. El resultado psicológico del estilo colegial en los empleados, es la autodisciplina.

\section{Estilo gerencial Conflictivo}

Jiménez (2004, p.112) define este estilo de gerencia como "al darle mayor importancia a lo informal pone en tela de juicio toda la organización formal de la empresa, y sin darse cuenta promueve constantemente la ruptura entre sus colaboradores", dando como resultado la corrosión de los cimientos de dicha organización en términos de clima organizacional. Se puede conceptualizar el estilo gerencial conflictivo, como aquel capaz de resolver de manera oportuna las diferencias o luchas de poder originadas por las directrices emanadas por su forma de planificar, organizar, dirigir y controlar al personal.

\section{Metodología}

En la presente investigación se implementó una metodología cuantitativo con análisis exploratorio y descriptivo. Del mismo modo las variables analizadas fueron definidas bajo la teoría fundamentada identificadas bajo la dimensión de los estilos gerenciales; los indicadores autocrático, democrático, consultivo, participativo, deliberado, colegiado, resolutivo y conflictivo. Dentro de los Participantes, se seleccionaron por conveniencia 28 empresas de diferentes sectores económicos; bajo la técnica de encuesta se sustrajo la información directamente de los gerentes de tales compañías. El instrumento fue diseñado de tipo encuesta, constituído por 12 preguntas; 8 de corte bimodales o dicotómicas y 4 politómicas, de ellas 3 con 6 opciones de respuesta y una con 5 opciones de respuesta.

El procedimiento investigativo se llevó a cabo en los meses comprendidos abril de 2017 hasta mayo 2017. El procedimiento estadístico se fundamentó en análisis de frecuencias ( $n$ ) y medidas de tendencia central en primera instancia (media-M) y medidas de dispersión (Desviación típica DT) 
Tabla 1

Instrumento de medida

\begin{tabular}{|c|c|c|c|}
\hline Variable & Ítem & Tipo Pregunta & Tipo Respuesta \\
\hline Autoridad & Ítem 1. En el área administrativa la autoridad es: & Politómica & 6 opciones R \\
\hline Gerencia & Ítem 2. En el área administrativa para los gerentes: & Politómica & 6 opciones $\mathrm{R}$ \\
\hline Democrático & $\begin{array}{l}\text { Ítem 3. ¿En el área administrativa el gerente decide conjuntamente con los trabajadores } \\
\text { cuál es la mejor alternativa a seguir? }\end{array}$ & Dicotómica & Sí / No \\
\hline Participativa & $\begin{array}{l}\text { Ítem } 4 \text {. En el área administrativa la gerencia propicia en los trabajadores una acción } \\
\text { participativa basada en el: }\end{array}$ & Politómica & 5 opciones $\mathrm{R}$ \\
\hline Consultivo & Ítem 5. En el área administrativa el gerente conjuntamente con sus subordinados formula: & Politómica & 6 opciones $\mathrm{R}$ \\
\hline Resolución de conflictos & $\begin{array}{l}\text { Ítem 6. ¿En el área administrativa los trabajadores participan conjuntamente con la } \\
\text { gerencia en la resolución de los problemas? }\end{array}$ & Dicotómica & Sí / No \\
\hline \multirow[t]{2}{*}{ Estilo-Participativo } & $\begin{array}{l}\text { Ítem 7. ¿En el área administrativa el gerente solamente se reserva el control, partiendo } \\
\text { del principio: "el control está en función de la responsabilidad y por lo tanto no es } \\
\text { delegable"? }\end{array}$ & Dicotómica & Sí / No \\
\hline & $\begin{array}{l}\text { Ítem 8. ¿En el área administrativa el gerente señala directrices específicas a sus } \\
\text { subalternos, pero consulta sus ideas y opiniones sobre muchas decisiones que les } \\
\text { incumben? }\end{array}$ & Dicotómica & Sí / No \\
\hline \multirow[t]{2}{*}{ Estilo-Colegiado } & $\begin{array}{l}\text { Ítem 9. ¿En el área administrativa las funciones se ejercen en conjunto, incluyendo el } \\
\text { control? }\end{array}$ & Dicotómica & Sí / No \\
\hline & $\begin{array}{l}\text { Ítem 10. ¿En el área administrativa se crea una estricta relación de mutualidad, en la cual } \\
\text { cada persona realiza sus propias contribuciones y aprecia la de los demás? }\end{array}$ & Dicotómica & Sí / No \\
\hline \multirow[t]{2}{*}{ Estilo Conflictivo } & $\begin{array}{l}\text { Ítem } 11 . \text { ¿En el área administrativa el gerente construye relaciones amistosas de manera } \\
\text { perspicaz? }\end{array}$ & Dicotómica & Sí / No \\
\hline & Ítem 12. ¿En el área administrativa los gerentes mantienen una lucha por el poder? & Dicotómica & Sí / No \\
\hline
\end{tabular}

Fuente: Elaboración propia

\section{Resultados}

A la luz de los resultados en la tabla 1, el 100\% de los encuestados manifestó que es descentralizada, por cuanto cada Gerencia estipula sus normas en virtud de los objetivos formulados por la organización, y a su vez, los trabajadores bajo su mando, al conocer los objetivos, políticas, metas, normas a seguir, planifican y programan sus actividades, desplegándose así, desde la cúpula o Gerencia General los parámetros a alcanzar en el tiempo estipulado para ello, y en función a esto, tanto los Gerentes de línea como los Coordinadores se organizan de manera descentralizada para garantizar su consecución.

Tabla 2

Frecuencias de las variables

\begin{tabular}{|l|r|r|}
\hline \multicolumn{3}{|c|}{ Autoridad } \\
\hline \multirow{2}{*}{ Ítem 1. En el área administrativa la autoridad es: } \\
\hline Unilateral & \multicolumn{2}{|l|}{ Frecuencia } \\
\cline { 2 - 4 } & Fa & Fr (\%) \\
\hline Descentralizada & 0 & 0 \\
\hline Reciprocidad & 0 & $100 \%$ \\
\hline Participativa & 0 & 0 \\
\hline Todas las anteriores & 0 & 0 \\
\hline Ninguna de las anteriores & 0 & 0 \\
\hline Totales & $\mathbf{2 8}$ & $\mathbf{1 0 0} \%$ \\
\hline \multicolumn{2}{|c}{} \\
\hline \multicolumn{2}{|c|}{ Participativa } & \\
\hline
\end{tabular}

Ítem 4. En el área administrativa la gerencia propicia en los trabajadores una acción participativa basada en el:

\begin{tabular}{|l|r|r|}
\hline \multirow{2}{*}{ TR } & \multicolumn{2}{|l|}{ Frecuencia } \\
\cline { 2 - 3 } & Fa & Fr (\%) \\
\hline Consenso & 28 & $100 \%$ \\
\hline Negociación & 0 & 0 \\
\hline Persuasión & 0 & 0 \\
\hline Todas las anteriores & 0 & 0 \\
\hline Ninguna de las anteriores & 0 & 0 \\
\hline Totales & $\mathbf{2 8}$ & $\mathbf{1 0 0 \%}$ \\
\hline
\end{tabular}

\begin{tabular}{|c|c|c|}
\hline \multicolumn{3}{|l|}{ Gerencia } \\
\hline \multirow{2}{*}{ TR } & \multicolumn{2}{|l|}{ Frecuencia } \\
\hline & $\mathrm{Fa}$ & Fr (\%) \\
\hline Los trabajadores sólo cumplen órdenes & 0 & 0 \\
\hline Los trabajadores pueden aportar ideas & 28 & $100 \%$ \\
\hline Los trabajadores sólo son responsables por las tareas asignadas & 0 & 0 \\
\hline No pueden ejecutar tareas que no han sido asignadas & 0 & $\overline{0}$ \\
\hline Todas las anteriores & 0 & 0 \\
\hline Ninguna de las anteriores & 0 & 0 \\
\hline Totales & 28 & $100 \%$ \\
\hline \multicolumn{3}{|l|}{ Consultivo } \\
\hline \multicolumn{3}{|c|}{$\begin{array}{l}\text { Ítem } 5 \text {. En el área administrativa el gerente conjuntamente con sus subordinados } \\
\text { formula: }\end{array}$} \\
\hline \multirow{2}{*}{ TR } & \multicolumn{2}{|l|}{ Frecuencia } \\
\hline & $\mathrm{Fa}$ & Fr (\%) \\
\hline Metas & 0 & 0 \\
\hline Objetivos & 28 & $100 \%$ \\
\hline Planes & 0 & 0 \\
\hline Políticas & 0 & 0 \\
\hline Todas las anteriores & 0 & 0 \\
\hline Ninguna de las anteriores & 0 & 0 \\
\hline Totales & 28 & $100 \%$ \\
\hline
\end{tabular}

Fuente: Elaboración propia 
Por otro lado, el $100 \%$ de los encuestados escogió la opción donde se establece que los trabajadores pueden aportar ideas, por cuanto para la organización lo más importante es aprovechar las habilidades, destrezas, experiencias e intuición de los trabajadores manifestadas a través de sus ideas, las cuales, generan matriz de opiniones, además de escenarios claros sobre las situaciones a abordar con celeridad, pudiendo así dirimir sobre la alternativa más favorable, realista y viable a seguir, en la consecución eficaz y eficiente de los objetivos o en la resolución de los problemas.

Con relación a la Acción Participativa, el 100\% de los encuestados indicó el consenso, por cuanto tiene que estar la mayoría de acuerdo con las directrices a seguir a fin de crear un clima de armonía donde cada trabajador sea capaz de desarrollar al máximo sus habilidades, destrezas, ideas, experiencias en pro de la consecución eficaz y eficiente de los objetivos planteados para el corto, mediano, largo plazo.

Tabla 3

Frecuencias de las variables

\begin{tabular}{|c|c|c|c|c|c|c|c|c|c|c|c|c|c|c|c|c|}
\hline \multirow[t]{2}{*}{ T.R } & \multicolumn{2}{|c|}{ I3 Est-Democrático } & \multicolumn{2}{|c|}{ I6 Resol. conflictos } & \multicolumn{4}{|c|}{ 17-I8 Estilo-Participativo } & \multicolumn{4}{|c|}{ I9-I10 Estilo-Colegiado } & \multicolumn{4}{|c|}{ I11-I12 Estilo Conflictivo } \\
\hline & $\mathrm{Fa}$ & $\operatorname{Fr}(\%)$ & $\mathrm{Fa}$ & $\operatorname{Fr}(\%)$ & $\mathrm{Fa}$ & Fr (\%) & $\mathrm{Fa}$ & $\mathrm{Fr}(\%)$ & $\mathrm{Fa}$ & $\mathrm{Fr}(\%)$ & $\mathrm{Fa}$ & $\operatorname{Fr}(\%)$ & $\mathrm{Fa}$ & $\operatorname{Fr}(\%)$ & $\mathrm{Fa}$ & $\operatorname{Fr}(\%)$ \\
\hline Si & 12 & $43 \%$ & 16 & $57 \%$ & 0 & $0 \%$ & 28 & $100 \%$ & 28 & $100 \%$ & 28 & $100 \%$ & 0 & $0 \%$ & 0 & $0 \%$ \\
\hline No & 16 & $57 \%$ & 12 & $43 \%$ & 28 & $100 \%$ & 0 & $0 \%$ & 0 & $0 \%$ & 0 & $0 \%$ & 28 & $100 \%$ & 28 & $100 \%$ \\
\hline Total & 28 & $100 \%$ & & $100 \%$ & 28 & $100 \%$ & 28 & $100 \%$ & 28 & $100 \%$ & 28 & $100 \%$ & 28 & $100 \%$ & 28 & $100 \%$ \\
\hline
\end{tabular}

Fuente: Elaboración propia

Por otra parte en la tabla 3, se visualiza que el 57\% de los encuestados optó por el no, por cuanto la gerencia a la cual están ellos adscritos no admite su intervención en el proceso decisorio, sin embargo, el 43\% restante manifestó sí participar cuando se escoge la mejor alternativa a seguir porque los gerentes con quien ellos trabajan si les permiten interactuar y formar una matriz de opiniones de donde se deslinda la alternativa a seguir, de esta manera, queda evidenciado que en el área administrativa existen menos gerentes receptivos al estilo democrático, con la participación de los empleados en la toma de decisiones, mientras que la mayoría no lo son.

Sin embargo el 57\% de los encuestados aludió al sí en la resolución de conflictos, por cuanto para los gerentes sus ideas son muy importantes para la formulación de una matriz de opiniones capaz de reflejar las alternativas viables, objetivas, reales y oportunas para asumir con celeridad la solución eficiente y eficaz de los problemas. No obstante, el $43 \%$ restante indicó que ellos no tienen inherencia en la resolución de los problemas, solo se limitan a solventar las situaciones adversas que tengan relación directa con las tareas a ejecutar, pero si no es trascendental dicho problema.

\section{Gráfico 1}

Frecuencias de las variables

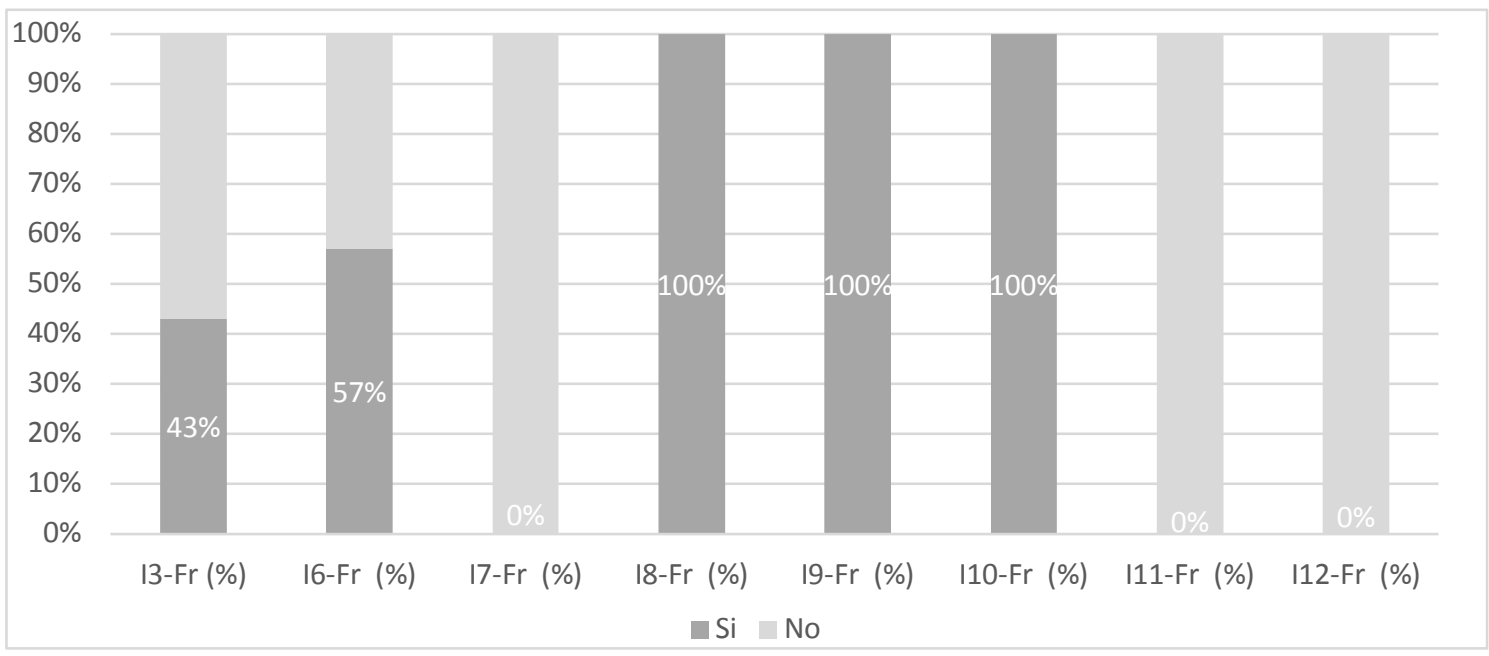

Fuente: Elaboración propia 
Frente al estilo participativo, el $100 \%$ de los encuestados comentó que no, por cuanto todos en virtud de sus funciones tienen que administrar el control inherente a la actividad que ejecuta, así, todos son co- responsables de los resultados obtenidos, debiendo comunicar cómo, cuándo, y por qué se solventaron las desviaciones registradas, además de quienes intervinieron en dicha actuación.

Por otro lado, al indagar sobre, ¿En el área administrativa el gerente señala directrices específicas a sus subalternos, pero consulta sus ideas y opiniones sobre muchas decisiones que les incumben?, el $100 \%$ de los encuestados indicó que si, por ser esto una garantía de incentivar el sentido de pertenencia, de compromiso con la organización por sentirse tomados en cuenta, responsables y participes de todo lo relacionado con las tareas a desarrollar, para la consecución de los objetivos previstos para el corto, mediano, largo plazo.

Lo reflejado en la tabla 3 y gráfico 1, muestra al preguntar, ¿En el área administrativa las funciones se ejercen en conjunto, incluyendo el control?, que el $100 \%$ de los encuestados manifestó que si, por garantizar ello el logro eficiente y eficaz de los objetivos organizacionales, pero no se toman en conjunto las decisiones.

Asimismo, al indagar sobre, ¿En el área administrativa se crea una estricta relación de mutualidad, en la cual cada persona realiza sus propias contribuciones y aprecia la de los demás?, el 100\% respondió que sí, por ser esto lo único que garantiza el trabajo en equipo, responsable, objetivo, real, veraz, todos deben trabajar siguiendo las mismas directrices suministradas de manera clara y oportuna, todos deben respetar los aportes de cada persona, porque con ellos se crea la matriz de opiniones tan valiosa para la resolución de problemas y toma de decisiones.

Con base a lo reflejado en la tabla 3 y gráfico 1, al preguntar, ¿En el área administrativa el gerente construye relaciones amistosas de manera perspicaz?, se visualiza que el $100 \%$ de los encuestados escogió el no, por cuanto las relaciones amistosas se erigen bajo la premisa de la confianza, respeto, y responsabilidad en la forma de actuar, quedando eso claramente establecido cuando se llega a la organización o cuando las personas son trasladadas de un puesto a otro.

Por otro lado, al indagar sobre, ¿En el área administrativa los gerentes mantienen una lucha por el poder?, el $100 \%$ de los encuestados optó por el no, fundamentado en el hecho de que la lucha de poder sólo origina conflictos y estos inciden de manera negativa en la consecución de los objetivos organizacionales, productividad, rentabilidad.

\section{Discusión}

Los hallazgos obtenidos en el presente estudio confirman que el estilo democrático es ausente en la mayoría de las empresas encuestadas, sigue prevaleciendo la autocracia en la gerencia. Se confirma presencia del estilo de resolución de conflictos en la mayoría de las empresas, aunque el porcentaje menor al anterior deja en entre dicho si este estilo está bien definido por las empresas teniendo en cuenta su baja diferencia; 4,3 de cada 10 empresas, los trabajadores no participan en la resolución de problemas junto con la gerencia.

Además se encontró en la investigación que los estilos gerenciales Participativo y Colegiado están presentes en todas las empresas, esto conversa con los resultados del estudio de Hernández et al. (2017), resultados que son muy positivos para la sana administración de las compañías, generación de valor y clima organizacional, este estilo proyecta seguridad y confianza en los colaboradores según Ruiz (2012); el estilo conflictivo es ausente y contribuye del mismo modo a la convivencia y sana competitividad de los colaboradores.

Son varios los trabajos que han puesto previamente de manifiesto esta relación (Galindo, 2012, p.99; González, 2014, p.101; Goncalves, 2010, p.107; Hernández et al., 2017; Jiménez, 2004, p.102; Mora, 2014; Solys et al., 2016; Soto, 2012; Suárez \& Pinzón, 2020); existió concordancia en los trabajos expuestos en el marco teórico y los resultados obtenidos; aunque, no se hayó concordancia con el planteamiento de (Bateman, 2011, p.110; Cenzo, 
2012, p.32; Villava \& Fierro, 2017) en el estilo democrático, y las variables de Autoridad y Gerencia con relación al estilo autocrático.

Según la teoría fundada y los resultados optenidos, el presente trabajo tiene como significado que las altas direcciones de las empresas son ambigúas en los diferentes estilos gerenciales, si bien anún no están aplicados todos los estilos al 100\% favorablemente, hay una alta tasa de probabilidad que la gerencia en las 28 empresas encuestadas hacen una gerencia idónea en función de los colaboradores y área administrativa. Existen ciertos comportamientos en los gerentes dejados en su reserva naturalmente como el Neuroliderázgo que tiene en cuenta las emociones (Suárez \& Pinzón, 2020), los gerentes no "sueltan las riendas" del todo de sus cargos en los colaboradores administrativos, su responsabilidad hace que los gerentes tengan un acercamineto y control constante en las actividades, desiciones y objetivos a lograr conincidiendo con el estudio de Solys et al. (2016). Existe el riesgo de perder el horizonte de sus compañías en la medida que no haya una vigilancia controlada en sus compañías, dejando en entre ver su autocracia y dirección permanente ante sus subordinados.

Finalmente se encontraron limitantes dentro del trabajo de campo que no permitieron tener profundidad del estudio, como lo fue la baja participación de las empresas; el tiempo límite para la resolución del cuestionario; conocimiento a profundidad de los procesos y porcedimientos internos con relación a la incidencia por cada administrativo para la toma de desiciones; y el limitado suministro de información de las epresas participantes.

\section{Conclusiones}

Una vez efectuada la investigación donde se propuso el empowerment como estilo gerencial se concluye que efectivamente en las empresas se da el empoderamiento en cierta medida, teniendo en cuenta que a los trabajadores se les proporciona un nivel moderado de democracia y autorización desentralizada. Asimismo, se propicia en los trabajadores la acción participativa basada en el consenso, negociación y persuasión, a fin de lograr la colaboración de todos los involucrados tanto en la realización como en la resolución de problemas que se puedan suscitar.

El trabajo investigativo logra los objetivos propuestos inicialmente planteados, identificando claramente los términos relacionados con el empoderamiento en las organizaciones y los diferentes estilos gerenciales que dan apertura al empoderamiento del área administrativa de la compañía. En este sentido desde la gerencia se brinda confianza en los colaboradores para la toma de desiciones, resolución de conflictos y participación en las desiciones relevantes de las empresas; sin delegar del todo el control por tener representación en el estilo autocrático, de igual forma se ejersen las funciones en conjunto, incluyendo el control para crear una estricta relación de mutualidad en donde cada trabajador emite sus propias contribuciones, apreciando la de los demás y evitando de esta manera la construcción de relaciones amistosas perspicaces por parte de la gerencia y la lucha por el poder.

En este sentido, quedó de manifiesto el estilo gerencial como democrático, consultivo, participativo y colegiado, donde impera la premisa tendiente a la formación de equipos de trabajo capaces de auto gestionarse para trabajar en conjunto con su jefe inmediato, a fin de alcanzar de manera eficiente y eficaz los objetivos fijados para el corto, mediano y largo plazo. Se propone para futuras investigaciones aondar en especificdades que dan cuenta en la desición sobre procesos y procedimientos de cada área, además de la aplicación de un instrumento que evalúe las percepciones específicas de los subordinados en comparativas con otras empresas de otros sectores con el fin de crear constructos determinantes que complemente el estudio científico del fenómeno; de esta forma y con la expansión del estudio aplicado a otros países de latinoamérica podría enriquecer el conocimiento generalizando un comportamiento específico de los países latinoamericanos. 


\section{Refrencias bibliográficas}

Antoncic, B., \& Hisrich, R. D. (2001). Intrapreneurship: Construct Refinement and Cross-Cultural Validation. Journal of Business Venturing, 16(5), 495-527. doi:10.1016/S0883-9026(99)00054-3

Blanchard, K.; Carlos, J. P. y Randolph, A. (2009). Empowerment: tres claves para el proceso de facultar a los empleados funcionen en su empresa. Bogotá: Grupo Editorial norma.

BP-I, (2013). Estilos gerenciales que se pueden aplicar a tu pyme. [Mensaje en un blog]. Recuperado de https://www.impulsapopular.com/gerencia/estilos-gerenciales-que-se-pueden-aplicar-a-tu-pyme/

Bateman, T. y Snell, S. (2006). Administración una ventaja competitiva. México. D.F: Editorial Mc. Graw Hill.

Calderón, H. G., (2003). Dirección de recursos humanos y competitividad. Revista Innovar 22, 157-172.

Codina, A. (2012). El gerente de hoy en una empresa exitosa. (En línea) Colombia

Chiavenato, I., (2007). Administración de Recursos Humanos. México. McGraw Hilll

Chiavenato, I., (2011). Administración de Recursos Humanos. Santa Fe Bogotá McGraw Hilll. Colección General.

Chiavenato, I. (2012). Administración de recursos humanos. México. Editorial Mc Graw Hill

De la Garza, C. T., Carpio, M., López, J. y Rodríguez, P. (2020). El liderazgo de servicio en relación al involucramiento en el trabajo. Revista Espacios 4(8) 39-40. Recuperado de https://bit.ly/2ZXhm5L

De Villiers-Scheepers, M. J. (2012). Antecedents of Strategic Corporate Entrepreneurship. European Business Review, 24(5), 400-424. doi:10.1108/09555341211254508

Díaz, (2009). Definición del Empowerment: Grupo Editorial norma.

Drucker, P. (2012). Las Nuevas Realidades. Bogotá, Colombia: Editorial Norma, Santa Fe de Bogotá

Do Nascimento, S., \& Beuren, I. M. (2015). A eficácia gerencial aferida pelo alcance das metas estabelecidas no sistema de recompensa e sua relação com o empowerment psicológico. Innovar, 25(55), 59-73. http://dx.doi.org/10.15446/innovar.v25n55.47196.

Galván Vela, E., \& Sánchez Limón, L. (2018). Factores organizacionales relacionados con el comportamiento intraemprendedor. Innovar, 29(71), 55-68. https://doi.org/10.15446/innovar.v29n71.76395.

Goncalves, A. (2010). Fundamentos del clima organizacional. México, DF. Sociedad latinoamericana para la calidad (SLC).

González, M. (2014). Comportamiento organizacional un enfoque latinoamericano. México, DF. Compañía edit. Continental.

Harvard Deusto Business Review y EAE Business School (2017). Características principales del liderazgo participativo. Recuperado el 19 Febrero del 2017, de https://bit.ly/20hhk3a

Hernández, H., Muñoz, D. \& Barrios, I. (2017). Estilos gerenciales y su influencia en la generación de valor de las instituciones prestadoras de salud de la región Caribe. Económicas CUC, 38(1), 133-146.

http://dx.doi.org/10.17981/econcuc.38.1.06

Illanes, J. y Esparza P. (2015). La gestión Participativa: Un enfoque para la moderna dirección de las organizaciones. Universidad Católica Raúl Silva Hernández. Tesis de Grado. Distrito Federal- México 
Jiménez, A. R. (2004). Tipos de Liderazgo. Recuperado de https://bit.ly/329m0jQ. Consultado el: 10/04/2017.

Kreitner, R.y Kinicki, A (2018). Comportamiento Organizacional. México: Editorial Mc. Graw Hill. Interamericana.

Lewin, K. (1951). Field theory of science: Selected theorical chapter. The annals of the American academy of political and social science. 276(1), 146-147. Doi: 10.1177/000271625127600135

Meriño, C. V.; Martínez, de M. C.; Ortiz, S. L.; Trejos-Gil, C.; López-Giraldo, J. \& Beltrán-Ríos, J. (2020). Sistema axiológico de los alumnos del Programa Contaduría Pública de la Universidad Católica Luis Amigó Medellín-Colombia. Revista Espacios 14(16) 12-18. Recuperado de: https://bit.ly/36JScdN

Mora, J. (2014). Estilos gerenciales. [Mensaje en un blog]. Recuperado de: https://bit.ly/3iTstoU

Prieto, R., Burgos, C., García, J. y Rincón, Y. (2016). Mercadeo interno para optimizar la calidad de servicio en la banca universal. Revista venezolana de gerencia, 21(73) 102-119

Robbins S. P. y Coulter M. (2010). Administración. 5 ed. México, DF. Edit. Prentice Hall.

Ruiz, P. A. (2012). Las Habilidades Gerenciales y su Importancia en la Dirección Empresarial. Revista MyM

Recuperado de: https://bit.ly/2UJVgSh.

Shenav, Y. (1995). From chaos to systems: The engineering foundations of organization theory. Administrative Science Quarterly 40(3) 557-585.

Solys, G. A. P., Morales, M. B., y Medina, W. M. G. (2016). Los estilos de liderazgo y su influencia en el desarrollo empresarial: caso Pymes de la provincia de Tungurahua-Ecuador. Revista PUCE 103, 355-369. Recuperado de http://www.revistapuce.edu.ec/index.php/revpuce/article/view/45/200

Soto, B. (2012). El liderazgo democrático. [Mensaje en un blog]. Recuperado de: https://bit.ly/329hFwW

Suárez, R. y Pinzón, F., (2020). Neuroliderazgo: Simulación de un escenario como parte del proceso de selección Organizacional. Espacios 41(8) 17-26. Recuperado de https://bit.ly/3fkZKao

Trejos-Gil, C. A. (2008). El marketing directo en la organización en pro de la construcción de marca. Revista de Investigaciones Universidad Católica de Manizales, 12(8), 84-94. Recuperado de: https://bit.ly/2M8I19d

Villava, M. y Fierro, I. (2017) El liderazgo Democrático: Una aproximación conceptual. INNOVA Research Journal 2 (4) $155-162$

Vroom, V. y Yetton, P. (1973). Leadership and decision making. Pittsburgh: University of Pittsburgh Press.

Werther, W. y Davis, K. (2013). Administración de personal y recursos humanos. México, DF. Edit. McGraw-Hill.

Esta obra está bajo una Licencia Creative Commons Attribución-NoCommercial 4.0 International

$(\mathrm{cc})$ BY-NC 